\title{
Gauge Invariant Wave Equations in Curved Space-Times and Primordial Magnetic Fields
}

\author{
G. Lambiase ${ }^{a, b}$ and A.R. Prasanna ${ }^{c}$ \\ ${ }^{a}$ Dipartimento di Fisica "E.R. Caianiello", Universitá di Salerno, 84081 Baronissi (Sa), Italy. \\ ${ }^{b}$ INFN - Gruppo Collegato di Salerno, Italy. \\ ${ }^{c}$ Physical Research Laboratory, Navrangpura, Ahmedabad 380 009, India.
}

(August 28, 2018)

\begin{abstract}
The inflationary production of magnetic field seeds for galaxies is discussed. The analysis is carried out by writing the wave equation of the electromagnetic field in curved spacetimes. The conformal invariance is broken by taking into account of the interaction of the electromagnetic field with the curvature tensor of the form $\lambda R_{\alpha \beta \gamma \delta} F^{\alpha \beta} F^{\gamma \delta}$. Such a term induces an amplification of the magnetic field during the reheating phase of the universe, but no growth of the magnetic field occurs in the de Sitter epoch. The resulting primordial magnetic field turns out to have strengths of astrophysical interest.
\end{abstract}

PACS No.: 98.80.Cq, 98.62.En

One of the open issue of modern astrophysics is the origin of intragalactic magnetic fields, with the characteristics that their amplitudes are of the order of $\sim 10^{-6} \mathrm{G}$ and they are uniform on scales of the order of $\sim 10 \mathrm{kpc}$.

A promising candidate to explain the primordial magnetic field generation is the dynamo mechanism discussed by Zeldovich, Ruzmaikin and Sokoloff [1], and by Parker [2]. The dynamo effect induces an amplification of a preexisting magnetic field, but it requires a seed field at the epoch of galaxy formation coherent over a scale of $\sim 1 \mathrm{Mpc}$. The question that arises is about the mechanism that produced such a seed field.

Starting with the observation that the Universe, during its evolution, has behaved as a good conductor, one expects that the evolution of a primordial magnetic field may preserve the magnetic flux. This physical aspect is encoded in the parameter $r=\rho_{B} / \rho_{\gamma}$, which remains (with good approximation) constants and provides an invariant measure of magnetic field strength. Here $\rho_{B}=|\mathbf{B}|^{2} / 8 \pi$ is the energy density of a magnetic field, and $\rho_{\gamma}=\pi^{2} T^{4} / 25$ is the energy density of the cosmic microwave background radiation. In order to explain the present value of $r \approx 1$ for galaxies, one needs a pre-galactic magnetic field to which corresponds (see for example, Refs. [3,4])

$$
r \simeq 10^{-34} .
$$

Turner and Widrow have suggested that a magnetic field might be generated by quantum fluctuations during an inflationary epoch, and it could be sustained after the wave length of interest crossed beyond the horizon giving the observed field today [3]. This model invokes a coupling among the electromagnetic field and the curvature tensors $R$ and $R_{\alpha \beta}$, which break the conformal invariance. Nevertheless the field equations derived in Ref. [3] are not gauge invariant. Turner-Widrow's model has been generalized by Garretson, Field, and Carroll [5] by introducing a coupling of the photon with an arbitrary pseudo Goldstone boson (see also [6]). Ratra introduced a coupling between the scalar field $\phi$ and the electromagnetic field via the interaction $e^{\phi} F_{\mu \nu} F^{\mu \nu}$, where $F_{\mu \nu}$ is the electromagnetic field strength, $F_{\mu \nu}=\nabla_{\mu} A_{\nu}-\nabla_{\nu} A_{\mu}$. [7]. The generation of primordial magnetic field induced by coupling terms of the form $R^{n} F_{\mu \nu} F^{\mu \nu}$, which preserves the gauge invariance, has been studied by Mazzitelli and Spedalieri [4], whereas Dolgov has studied the breaking of conformal invariance in QED due to trace anomalies [8]. For other mechanisms aimed to explain in what way the primordial magnetic field might be generated, see Refs. [9-20].

This paper concerns the origin of the primordial magnetic field in the framework of the non-minimal coupling of electromagnetic field in curved space time, whose action is given by [21,22]

$$
S=\int d^{4} x \sqrt{-g}\left(-\frac{1}{4} F_{\mu \nu} F^{\mu \nu}+\lambda R^{\mu \nu \rho \sigma} F_{\mu \nu} F_{\rho \sigma}\right) .
$$

where the $\lambda$-term, although invariant under CPT and general coordinate transformations, violates the Einstein's equivalence principle. The motivation to introduce the nonminimal coupling of electromagnetic field with the gravitational field [22] was essentially to understand the intricate relation between curvature and all forms of energy and their variations. Though at the present epoch, this coupling could be very small for cosmological background $\left(\lambda<10^{11} \mathrm{~cm}^{2} \sim 2.5 \times 10^{20} \mathrm{eV}^{-2}[21]\right)$, at the very early universe the effect of the nonminimal terms could become important, apart from breaking the conformal invariance which indeed is necessary for the generation of large enough magnetic seed from the vacuum. The fact that this lagrangian preserves the gauge invariance but breaks the conformal invariance and gets very small at the present epoch is indeed a very satisfactory situation. In our approach of 
writing the wave equation for the fields [23], we get additional contributions from the curvature terms apart from the nonminimal terms that have been shown to arise from one loop quantum corrections [24], indicating the qualitative significance of such a coupling in the early universe scenario.

Variation with respect to the four-potential $A_{\mu}$ yields the field equations [21]

$$
\nabla_{\alpha} F^{\alpha \mu}=-2 \lambda\left[R^{\mu \nu \rho \sigma} \nabla_{\nu} F_{\rho \sigma}+\left(\nabla_{\rho} R_{\sigma}^{\mu}\right) F^{\rho \sigma}\right] .
$$

We also note that the electromagnetic field $F_{\mu \nu}$ satisfies the Bianchi identities

$$
\nabla_{\mu} F_{\nu \lambda}+\nabla_{\nu} F_{\lambda \mu}+\nabla_{\lambda} F_{\mu \nu}=0 .
$$

Eqs. (3) and (4) give the Maxwell equations in curved spacetimes. To infer the wave equations for $F_{\mu \nu}$, we apply the covariant derivative $\nabla_{\lambda}$ to Eq. (3), and by making use of Eq. (4) we get

$$
\square F_{\nu \lambda}+\left[\nabla^{\mu}, \nabla_{\nu}\right] F_{\lambda \mu}-\left[\nabla^{\mu}, \nabla_{\lambda}\right] F_{\nu \mu}=-2 \lambda\left(\nabla_{\lambda}\left[R^{\mu \nu \rho \sigma}\left(\nabla_{\nu} F_{\rho \sigma}\right)\right]+\nabla_{\lambda}\left[\left(\nabla^{\rho} R_{\lambda}{ }^{\sigma}\right) F_{\rho \sigma}\right]\right),
$$

where $\square=\nabla^{\mu} \nabla_{\mu}$ and [.,.] is the commutator. The Ricci identity [25]

$$
\left[\nabla^{\mu}, \nabla_{\nu}\right] F_{\alpha \mu}=R_{\rho \alpha \nu \mu} F^{\rho \mu}+R_{\nu}^{\rho} F_{\alpha \rho}
$$

and the well known cyclic identities of Riemann's tensor

$$
R_{\rho \alpha \beta \gamma}+R_{\rho \gamma \alpha \beta}+R_{\rho \beta \gamma \alpha}=0
$$

allow to cast Eq. (5) in the form

$$
\square F_{\nu \lambda}+R_{\nu \lambda}^{\rho \mu} F_{\rho \mu}+R_{\nu}^{\rho} F_{\lambda \rho}-R_{\lambda}^{\rho} F_{\nu \rho}=2 \lambda\left(\nabla_{\nu}\left[R_{\lambda}^{\alpha}{ }_{\lambda}^{\rho \sigma}\left(\nabla_{\alpha} F_{\rho \sigma}\right)\right]+\nabla_{\nu}\left[\left(\nabla^{\rho} R_{\lambda}{ }^{\sigma}\right) F_{\rho \sigma}\right]-(\nu \leftrightarrow \lambda)\right) .
$$

We now observe that the necessary and sufficient condition to have a system of coordinates in which the metric is conformal to the Minkowski one is that the Weyl tensor $C_{\lambda \mu \nu \rho}$ vanishes. As a consequence, the Riemann tensor can be written in terms of the Ricci tensor and the scalar curvature $R$ [26], i.e. (in $N$ dimensions)

$$
R_{\lambda \mu \nu \rho}=\frac{1}{N-2}\left(g_{\lambda \nu} R_{\mu \rho}-g_{\lambda \rho} R_{\mu \nu}-g_{\mu \nu} R_{\lambda \rho}+g_{\mu \rho} R_{\lambda \nu}\right)-\frac{R}{(N-1)(N-2)}\left(g_{\lambda \nu} g_{\mu \rho}-g_{\lambda \rho} g_{\mu \nu}\right) .
$$

After some algebras, for conformally flat spacetimes $(N=4)$ Eq. (8) simplifies to the form

$$
\begin{aligned}
\square F_{\nu \lambda}-\frac{R}{3} F_{\nu \lambda} & =2 \lambda\left(\nabla_{\nu}\left[R_{\lambda}^{\alpha}{ }_{\lambda}^{\rho \sigma}\left(\nabla_{\alpha} F_{\rho \sigma}\right)\right]+\nabla_{\nu}\left[\left(\nabla^{\rho} R_{\lambda}{ }^{\sigma}\right) F_{\rho \sigma}\right]-(\nu \leftrightarrow \lambda)\right) \\
& \left.=2 \lambda\left[\nabla_{\nu}\left(R_{\lambda \sigma} \nabla_{\rho} F^{\rho \sigma}-R_{\alpha \sigma} \nabla^{\alpha} F_{\lambda}{ }^{\sigma}-\frac{R}{3} \nabla^{\alpha} F_{\alpha \lambda}\right)+\nabla_{\nu}\left[\left(\nabla^{\rho} R_{\lambda}{ }^{\sigma}\right) F_{\rho \sigma}\right)\right]-(\lambda \leftrightarrow \nu)\right] .
\end{aligned}
$$

The metric components of the spatially flat Friedman-Robertson-Walker cosmology are, in the conformal time,

$$
g_{\mu \nu}=a^{2}(\eta) \operatorname{diag}(1,-1,-1,-1),
$$

where $a(\eta)$ is the scale factor. The field strength tensor $F_{\mu \nu}$ has components

$$
F_{\mu \nu}=a^{2}(\eta)\left(\begin{array}{cccc}
0 & -E_{x} & -E_{y} & -E_{z} \\
E_{x} & 0 & B_{z} & -B_{y} \\
E_{y} & -B_{z} & 0 & B_{x} \\
E_{z} & B_{y} & -B_{x} & 0
\end{array}\right) .
$$

whereas Riemann's and Ricci's tensor components are

$$
\begin{gathered}
R_{k l}^{i j}=-\frac{\dot{a}^{2}}{a^{4}}\left(\delta_{k}^{i} \delta_{l}^{j}-\delta_{l}^{i} \delta_{k}^{j}\right), \quad R_{0 j}^{0 i}=-\frac{1}{a^{2}}\left(\frac{\ddot{a}}{a}-\frac{\dot{a}^{2}}{a^{2}}\right) \delta_{j}^{i}, \quad R_{0 k}^{i j}=0, \\
R_{j}^{i}=-\frac{1}{a^{2}}\left(\frac{\ddot{a}}{a}+\frac{\dot{a}^{2}}{a^{2}}\right) \delta_{j}^{i} \equiv-u(\eta) \delta_{j}^{i}, \quad R_{0}^{0}=-\frac{3}{a^{2}}\left(\frac{\ddot{a}}{a}-\frac{\dot{a}^{2}}{a^{2}}\right) \equiv-s(\eta), \quad R_{0}^{i}=0,
\end{gathered}
$$


where the dot stands for derivative with respect to the conformal time $\eta$. Observing that in the conformal metric (10), the LHS of Eq. (9) for the spatial component assumes the form

$$
\square F_{i j}-\frac{R}{3} F_{i j}=\frac{1}{a^{2}} \square_{\eta} F_{i j},
$$

where $\square_{\eta}$ is the D'Alambertian in the Minkowski spacetime, i.e. $\square_{\eta}=\eta^{\mu \nu} \partial_{\mu} \partial_{\nu}=\partial^{2} / \partial \eta^{2}-\nabla_{\eta}^{2}$, it follows that Eq. (9) becomes

$$
\square_{\eta} F_{i j}=2 \lambda\left\{-\frac{2 s}{3} \ddot{F}_{i j}+\left(u-\frac{s}{3}\right) \nabla_{\eta}^{2} F_{i j}+\left[5(s-u) \frac{\dot{a}}{a}-\dot{u}\right] \dot{F}_{i j}-6(s-u)\left(\frac{\dot{a}}{a}\right)^{2} F_{i j}\right\},
$$

where $s$ and $u$ have been defined in (13). In terms of the magnetic field and collecting the terms, Eq. (15) reads

$$
\left(1+\frac{4 \lambda}{3} s\right) \frac{\partial^{2}}{\partial \eta^{2}}\left(a^{2} \mathbf{B}\right)-\left[1+2 \lambda\left(u-\frac{s}{3}\right)\right] \nabla_{\eta}^{2}\left(a^{2} \mathbf{B}\right)-2 \lambda\left[5(s-u) \frac{\dot{a}}{a}-\dot{u}\right] \frac{\partial}{\partial \eta}\left(a^{2} \mathbf{B}\right)+12 \lambda(s-u)\left(\frac{\dot{a}}{a}\right)^{2}\left(a^{2} \mathbf{B}\right)=0 .
$$

Taking the spatial Fourier transform

$$
\mathbf{B}(\eta, \mathbf{k})=\int \frac{d^{3} \mathbf{x}}{2 \pi} e^{i \mathbf{k} \cdot \mathbf{x}} \mathbf{B}(\eta, \mathbf{x})
$$

and using the notation

$$
\mathbf{F}_{k}(\eta)=a^{2}(\eta) \mathbf{B}(\eta, \mathbf{k})
$$

Eq. (16) can be rewritten as

$$
\left(1+\frac{4 \lambda}{3} s\right) \ddot{\mathbf{F}}_{k}-2 \lambda\left[5(s-u) \frac{\dot{a}}{a}-\dot{u}\right] \dot{\mathbf{F}}_{k}+\frac{n(\eta)}{\eta^{2}} \mathbf{F}_{k}=0,
$$

where

$$
n(\eta)=\eta^{2}\left\{\left[1+2 \lambda\left(u-\frac{s}{3}\right)\right] k^{2}+12 \lambda(s-u)\left(\frac{\dot{a}}{a}\right)^{2}\right\} .
$$

Let us now evaluate the magnetic field for the different phases of evolution of the Universe [3]. In what follows we concern with the evolutions of the magnetic field fluctuations whose wavelengths are well outside the horizon, according to [3], $L_{\text {phys }}=a L \gg H^{-1}$ or $k \eta \ll 1$. We shall use the notation $F_{k}=\left|\mathbf{F}_{k}\right|=\sqrt{\mathbf{F}_{k}^{*} \cdot \mathbf{F}_{k}}$.

Inflationary de Sitter $(d S)$ phase. The scale factor for this epoch of the Universe is

$$
a(\eta) \sim-\frac{1}{H_{d S} \eta}
$$

where $H_{d S} \sim 3 \times 10^{24} \mathrm{eV}$ [14]. Eq. (19) reduces to $\left(1+4 \lambda H_{d S}^{2}\right)\left(\ddot{F}_{k}+k^{2} F_{k}\right)=0$, whose solution is $F_{k}(\eta) \sim \sin k \eta$. It is worth to note that the $\lambda$-term does not affect the growth of the magnetic field. For modes outside the horizon, we have in fact that $F_{k}$ depends on $\eta$ as

$$
F_{k}(\eta) \sim \eta \sim a^{-1}
$$

Phase of Reheating (RH) and Matter Domination. The scale factor for this stage of the Universe is

$$
a(\eta) \sim \frac{1}{4} H_{0}^{2} R_{0}^{3} \eta^{2}
$$

where $R_{0} \sim 10^{26} h_{0}^{-1} \mathrm{~m}\left(0.6 \leq h_{0} \leq 0.8\right)$ is the present Hubble radius of the Universe, and $H_{0} \sim 100 h_{0} \mathrm{~km} / \mathrm{Mpc}$ sec is the Hubble parameter. The expressions for $u$ and $s$, defined in (13),

$$
u(\eta)=\frac{6}{b^{2}} \frac{1}{\eta^{6}}, \quad s(\eta)=-u(\eta) .
$$


where $b \equiv 16 \lambda /\left(H_{0}^{2} R_{0}^{3}\right)^{2}$, and the condition that the $\lambda$-term is dominant $\eta \ll b^{1 / 6}$, allow to rewrite Eq. (19) in the form

$$
\ddot{\mathbf{F}}_{k}-\frac{21}{\eta} \dot{\mathbf{F}}_{k}+\frac{72}{\eta^{2}} \mathbf{F}_{k}=0
$$

whose solution is

$$
F_{k}(\eta) \sim \eta^{18} \sim a^{9} .
$$

Eq. (25) shows that the magnetic field grows at very high power of scale factor $a$, which is the main amplification for the seed of primordial magnetic fields.

Phase of Radiation Domination (RD). The scale factor of the Universe is

$$
a \sim H_{0} R_{0}^{2} \eta
$$

Eq. (19) reduces

$$
\left(1-\frac{4 d}{\eta^{4}}\right) \ddot{\mathbf{F}}_{k}-\frac{32 d}{\eta^{5}} \dot{\mathbf{F}}_{k}-\frac{48 d}{\eta^{6}} \mathbf{F}_{k}=0
$$

where $d \equiv \lambda /\left(H_{0} R_{0}^{2}\right)^{2}$. The solution is

$$
F_{k}(\eta)=c_{1}^{\prime} \frac{12 d+5 \eta^{4}}{12 d \eta^{4}}+c_{2}^{\prime} \frac{20 d+3 \eta^{4}}{20 d \eta^{3}}
$$

Using as boundary condition $F_{k} \rightarrow$ constant for increasing conformal time, we get $c_{2}^{\prime}=0$. Hence the solution is $F_{k}(\eta) \sim 5 / 12 d+\eta^{-4}$. The last term is suppressed during the evolution of the universe thus $F_{k} \sim$ constant, which implies $\rho_{B} \sim a^{-4}$, as expected.

The expressions of $F_{k}(\eta)$ for different epochs of the evolution of the Universe allow to estimate the strength of the primordial magnetic field. According to Turner-Widrow's model [3], if one assumes that the Universe had gone through a period of inflation at GUT scale $\left(M_{G U T} \sim 10^{16} \div 10^{17} \mathrm{GeV}\right)$ and that fluctuations of the electromagnetic field have come out from the horizon where the Universe had gone through about 55 e-folding of inflation, then [3]

$$
r \approx\left(7 \times 10^{25}\right)^{-2(p+2)}\left(\frac{M_{G U T}}{m_{P l}}\right)^{4(q-p) / 3}\left(\frac{T_{R H}}{m_{P l}}\right)^{2(2 q-p) / 3}\left(\frac{T_{*}}{m_{P l}}\right)^{-8 q / 3} L_{M p c}^{-2(p+2)}
$$

where $T_{R H}$ is the reheating temperature, $T_{*}$ is the temperature at which plasma effects become dominant (i.e. the Universe first becomes a good conductor), and $m_{P l} \sim 10^{19} \mathrm{GeV}$ is the Planck mass. Finally, $p$ and $q$ are the exponents of the scale factor $a(\eta)$ during the $\mathrm{dS}$ and $\mathrm{RH}$ epochs which determine an increasing of the magnetic field. In our case $p=-1$ and $q=9$ (see Eqs. (22) and (25)). The temperature $T_{*}$ can be estimated via reheating processes [3] $T_{*}=\min \left\{\left(T_{R H} M_{G U T}\right)^{1 / 2} ;\left(T_{R H}^{2} m_{P l}\right)^{1 / 3}\right\}$, and for $T<T_{*} \rho_{B}$ evolves as $\rho_{B} \sim a^{-4}$. We note, however, that the reheating temperature $T_{R H}$ is given by $T_{R H}=\left\{10^{9} \mathrm{GeV}, M_{G U T}\right\}$. In Table I are reported the values of $r$. The range of variability of $r$ is $r \sim 10^{-50} \div 10^{-32}$, and we also note that because of the contribution of $\lambda$ the value for the seed field becomes extremely sensitive to the values of the physical parameters entering in $r$. In particular, $M_{G U T} \sim 10^{17} \mathrm{GeV}$, $T_{R H} \sim 10^{16} \mathrm{GeV}$, and $T_{*} \sim 10^{15.56} \mathrm{GeV}$ imply $r \sim 10^{-34}$.

In conclusion, the non-minimal coupling of the electromagnetic field with curvature terms, which breaks the conformal invariance but preserves the gauge invariance of the field wave equations, provides a scenario in which magnetic fields may be amplified. Such an growing occurs mainly during the reheating phase, whereas no amplification arises in the inflationary stage. This picture allows to infer the order of magnitude of $r$ necessary to generate the seed of galactic magnetic fields.

[1] Ya.B. Zeldovich, A.A. Ruzmaikin, D.D. Sokoloff, Magnetic Field in Astrophysics, Gordon and Breach, New York, 1983. 
TABLE I. Values of $r=\rho_{B} / \rho_{\gamma}$ at $1 \mathrm{Mpc}$ and for $M_{G U T} \sim 10^{16} \div 10^{17} \mathrm{GeV}, T_{R H}=\left\{10^{9}, 10^{15}-10^{17}\right\} \mathrm{GeV}$, and $T_{*}=\left\{10^{12}, 10^{15}, 10^{16}\right\} \mathrm{GeV}[3]$.

\begin{tabular}{llll}
\hline \hline$M_{\text {GUT }}(\mathrm{GeV})$ & $T_{R H}(\mathrm{GeV})$ & $T_{*}(\mathrm{GeV})$ & $r \sim 10^{-37}$ \\
$10^{17}$ & $10^{9}$ & $10^{12}$ & $10^{-33}$ \\
& $10^{15}$ & $10^{16}$ & $10^{-45}$ \\
& $10^{16}$ & $10^{15.56}$ & $10^{-34}$ \\
& $10^{16}$ & $10^{16}$ & $10^{-32}$ \\
$10^{16}$ & $10^{17}$ & $10^{12}$ & $10^{-50}$ \\
\hline \hline
\end{tabular}

[2] E.N. Parker, Cosmical Magnetic Field, Clarenden, Oxford, England, 1979.

[3] M.S. Turner, L.M. Widrow, Phys. Rev. D 37, 2743 (1988).

[4] F.D. Mazzitelli, F.M. Spedalieri, Phys. Rev. D 52, 6694 (1995).

[5] W.D. Garretson, G.B. Field, S.M. Carroll, Phys. Rev. D 46, 5346 (1992).

[6] S.M. Carroll, G.B. Field, R. Jackiw, Phys. Rev. D 41, 1231 (1990).

C. Corianó, Mod. Phys. Lett. A 47, 1253 (1992).

D. Harari, P. Sikivie, Phys. Lett. B 289, 67 (1992).

[7] B. Ratra, Astrophys. J. 391, L1 (1992).

[8] A.D. Dolgov, Phys. Rev. D 48, 2499 (1993).

[9] A.C. Davis, K. Dimopoulos, Phys. Rev. D 55, 7398 (1997).

K. Dimopoulos, Phys. Rev. D 57, 4629 (1998).

[10] T. Vashaspati, Phys. Lett. B 265, 258 (1991).

[11] T.W.B. Kibble, A. Vilenkin, Phys. Rev. D 52, 679 (1995).

J.T. Ahonen, K. Enqvist, Phys. Rev. D 57, 664 (1995).

[12] R. Opher, U.F. Wichoski, Phys. Rev. Lett. 78, 787 (1997).

[13] M. Gasperini, Phys. Rev. D 63, 047301 (2001).

[14] O. Bertolami, D.F. Mota, Phys. Lett. B 455, 96 (1999).

[15] E.A. Calzetta, A. Kandus, F.D. Mazzitelli, Phys. Rev. D 57, 7139 (1998).

[16] A.L. Maroto, Phys. Rev. D 64, 083006 (2001).

[17] M. Gasperini, M. Giovannini, G. Veneziano, Phys. Rev. Lett. 75, 3796 (1995).

[18] K. Enqvist, Int. J. Mod. Phys. D 7, 331 (1998).

[19] L.M. Widrow, Rev. Mod. Phys. 74, 331 (2002).

[20] D. Grasso, H.R. Rubinstein, Phys. Rep. 348, 163 (2001).

[21] A.R. Prasanna, S. Mohanty, Class. Quant. Grav. 20, 3023 (2003).

[22] A.R. Prasanna, Phys. Lett. A 37, 331 (1971).

A.R. Prasanna, Lett. Nuovo Cimento 6, 420 (1973).

A.J. Accioly, N.L.P Pereira de Silva, Progr. Theor. Phys. 76, 1179 (1986).

A.J. Accioly et al., Class. Quant. Grav. 14, 163 (1997).

[23] S. Mohanty, A.R. Prasanna, Nucl. Phys. B 526, 501 (1998).

[24] I.T. Drummond, S.J. Hathrell, Phy Rev D 22, 343 (1980).

[25] M. Carmeli, Classical Fields, General Relativity, and Gauge Theory, J. Wiley \& Sons, New York, 1982.

[26] S. Weinberg, Gravitation and Cosmology, J. Wiley \& Sons, New York, 1972. 